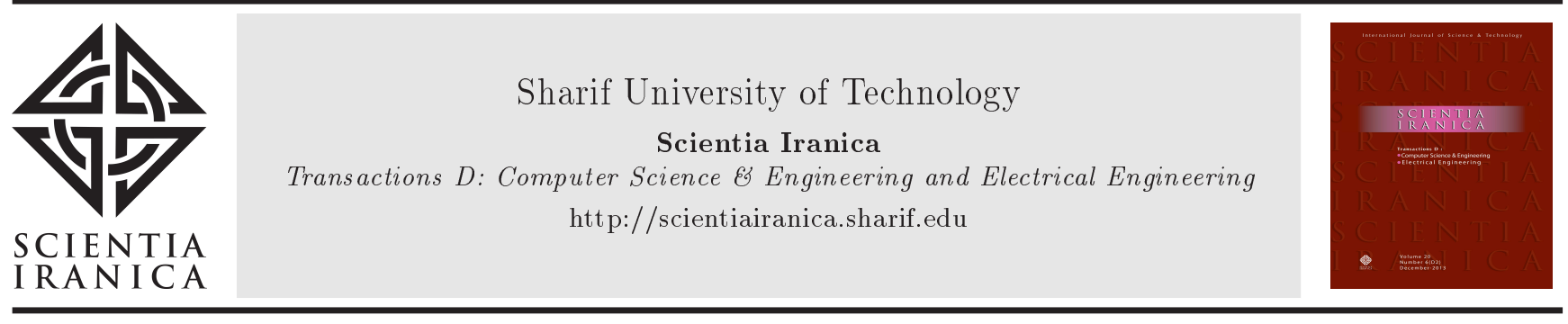

\title{
Reference tracking of nonlinear dynamic systems over AWGN channel using the describing function
}

\author{
A. Parsa and A. Farhadi* \\ Department of Electrical Engineering, Sharif University of Technology, Tehran, Iran.
}

Received 29 April 2017; received in revised form 20 August 2017; accepted 6 January 2018

\section{KEYWORDS}

Networked control system;

Nonlinear dynamic

system;

The describing

function.

\begin{abstract}
This paper presents a new technique for mean square asymptotic reference tracking of nonlinear dynamic systems over Additive White Gaussian Noise (AWGN) channel. The nonlinear dynamic system has periodic outputs and sinusoidal inputs and is cascaded with a bandpass filter acting as an encoder. By using the describing function method, the nonlinear dynamic system is represented by an equivalent linear dynamic system. Then, for this system, a mean square asymptotic reference tracking technique, including an encoder, a decoder, and a controller, is presented. It is shown that the proposed reference tracking technique results in mean square asymptotic reference tracking of nonlinear dynamic systems over the AWGN channel. The satisfactory performance of the proposed reference tracking technique is illustrated by practical simulations.

(C) 2019 Sharif University of Technology. All rights reserved.
\end{abstract}

\section{Introduction}

\subsection{Motivation and background}

One of the issues that has begun to emerge in a number of applications, such as tele-operation of micro autonomous vehicles [1] and smart oil drilling system [2], is how to stabilize a dynamic system over communication channels subject to imperfections (e.g., noise, packet dropout, distortion). This motivates research on stability problem of dynamic systems over communication channels subject to imperfections. Some results addressing the basic problems in stability of dynamic systems over communication channels subject to imperfections can be found in [3-20]. In most of these references, the goal is to construct reliable data and/or ensure the stability of (noisy) communication channels subject to limited capacity constraints. Intuitively, the results in this direction provide a quantitative under-

*. Corresponding author. Tel.: +982166165939 E-mail address: afarhadi@sharif.edu (A. Farhadi)

doi: $10.24200 /$ sci.2018.20108 standing of the way in which restriction on the data rate of the exchanged information among components of the system degrades the performance of the system. In another direction (e.g., [21]), the goal is to stabilize a dynamic system over communication channels subject to noise, delay, or loss and ensure no restriction on the transmission data rate.

Dynamic systems can be viewed as continuous alphabet information sources with memory. Therefore, many works in the literature (e.g., [3,9,10,16-20]) are dedicated to the problems of optimality and stability over Additive White Gaussian Noise (AWGN) channel, which is naturally a continuous alphabet channel [22]. Charalambous et al. [9,10] addressed the problem of mean square stability and tracking of linear Gaussian dynamic systems over AWGN channel when a noiseless feedback channel is available full time. In addition, they assumed that the communication of the control signal by a remote controller to the system was perfect. In [9], the authors presented an optimal control technique for asymptotic bounded mean square stability of a partially observed discrete-time linear Gaussian system over AWGN channel. In [10], the authors 
addressed the continuous time version of the problem addressed in [9] by focusing on the stability of a linear system with distinct real eigenvalues over SISI AWGN channel. In [16], the authors considered a framework for discussing control over a communication channel based on Signal-to-Noise Ratio (SNR) constraints and focused particularly on the feedback stabilization of an open-loop unstable plant via a channel with an SNR constraint. By examining the simple case of a linear time-invariant plant and an AWGN channel, they derived necessary and sufficient conditions of the SNR regarding feedback stabilization with an LTI controller. In [17], the authors presented a sub-optimal decentralized control technique for bounded mean square stability of a large-scale system with cascaded clusters of sub-systems. Each sub-system is linear and timeinvariant, and both subsystems and measurements are subject to Gaussian noise. The control signals are exchanged between sub-systems without any imperfections; however, the measurements are exchanged via an AWGN communication network. In [20], the authors investigated stabilization and performance issues of MIMO LTI networked feedback systems, in which the MIMO communication link was modeled as a parallel noisy AWN channel.

The above literature review reveals that the available results of the stability and tracking of dynamic systems over AWGN channel are concerned with linear dynamic systems and that there is no study result on the stability and reference tracking of nonlinear dynamic systems over AWGN channel. Nevertheless, many networked control systems, such as teleoperation system of autonomous vehicles [1] and smart oil drilling system [2], include nonlinear dynamic systems. This motivates research on the stability and reference tracking problems of nonlinear dynamic systems over the AWGN channel.

With respect to the role of nonlinear analysis and design in system analysis and design, several methods are available in the literature, including perturbation method, averaging method, harmonic balance method, etc. [23-25]. In addition, nonlinear analysis can be conducted in the frequency domain using, for example, the descending function method [26] and the Volterra series theory [27-29]. By using the Volterra series expansion, the Generalized Frequency Response Function (GFRF) was defined in [30], which is a multivariate Fourier transform of the Volterra kernels. This provides a useful concept for nonlinear analysis in the frequency domain, which generalizes the transfer function concept from linear systems to nonlinear systems. Moreover, a systematic method for nonlinear analysis, design, and estimation in the frequency domain is the nonlinear Characteristic Output Spectrum (nCOS). The nCOS function is an analytical and explicit expression for the relationship between nonlinear output spectrum and system characteristic parameters and can provide a significant insight into nonlinear analysis and design in the frequency domain $[27,31,32]$.

\subsection{Paper contributions}

In this paper, a new technique for reference tracking and stability of nonlinear dynamic systems over AWGN channel, as shown in Figure. 1, is presented. To achieve this goal, by using the describing function method, the equivalent linear dynamic system is extracted for a nonlinear dynamic system that has periodic outputs and sinusoidal inputs and is cascaded with a bandpass filter acting as an encoder. Then, we extend the result of [10] to account for the reference tracking of linear continuous-time systems with multiple real valued and non-real valued eigenvalues over Multi-Input, MultiOutput (MIMO) AWGN channel. Then, by applying these extended results to the equivalent linear dynamic system, mean square asymptotic reference tracking (and, hence, stability) of the nonlinear dynamic system over MIMO AWGN channel is addressed, as is shown in the block diagram of Figure 1. A similar block diagram concerning communication imperfections in a system and a remote controller has been considered in many research papers, such as [6,8-13]. This block diagram can correspond to the smart oil drilling system that uses down-hole telemetry [2] and, also, teleoperation systems of micro autonomous vehicles [1].

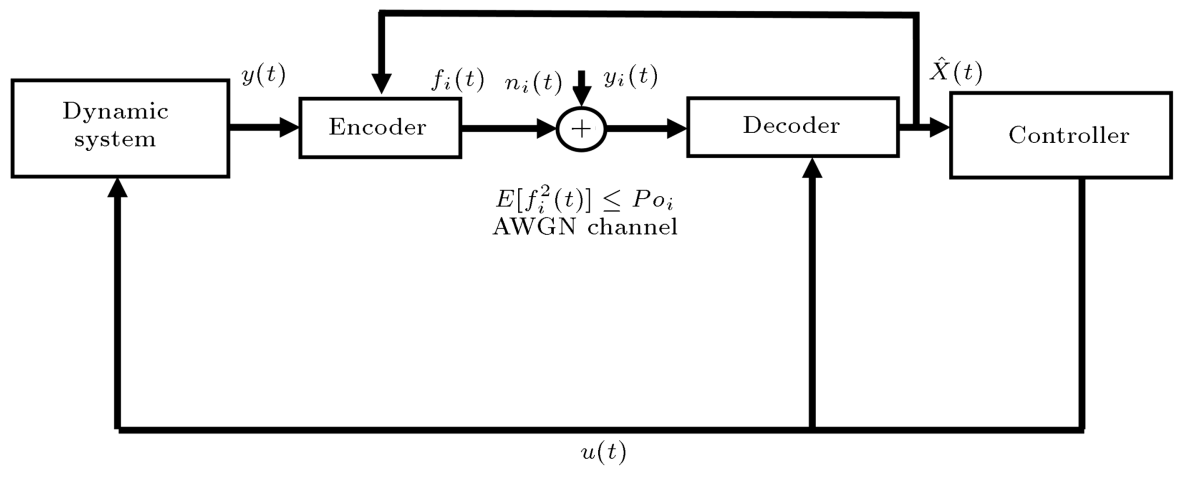

Figure 1. A dynamic system over MIMO AWGN channel. 
In the block diagram of Figure 1 , the encoder is a bandpass filter cascaded with a matrix gain; hence, the nonlinear dynamic system has a describing function as it has periodic outputs and sinusoidal inputs (e.g., the unicycle dynamic model [1], which can represent the dynamic of autonomous vehicles). That is, it can be represented by a linear dynamic system. In the field of nonlinear dynamic systems, the describing function is used for building oscillators [26]. However, in this paper, it is used to stabilize and control the nonlinear dynamic system in the block diagram of Figure 1. This is achieved in this paper by designing an encoder, a decoder, and a controller for the equivalent linear dynamic system that results in the asymptotic mean square tracking of the reference signal.

\subsection{Paper organization}

The paper is organized as follows. In Section 2, the problem formulation is presented. Section 3 is devoted to the describing function method. Then, in Section 4, the theory of mean square reference tracking of linear dynamic systems with multiple real valued and non-real valued eigenvalues over MIMO AWGN channel is developed. Section 5 is devoted to the mean square reference tracking of the nonlinear control system of Figure 1 over MIMO AWGN channel. Simulation results are given in Section 6, and the paper is concluded by summarizing the contributions of the paper and directions for future research in Section 7 .

\section{Problem formulation}

In this paper, certain conventions are used: $E[\cdot]$ denotes the expected value, $|\cdot|$ the absolute value, and $V^{\prime}$ the transpose of vector/matrix $V . A^{-1}$ denotes the inverse of a square matrix $A$, and $N(m, n)$ denotes the Gaussian distribution with mean $m$ and covariance $n$. $\mathbb{R}$ denotes the set of real numbers and $I_{n}$ the identity matrix with dimension $n$ by $n$, respectively. $\operatorname{trac}(A)$ denotes the trace of a square matrix, $A, \operatorname{diag}\{$.$\} denotes$ the diagonal matrix, $[A]_{i j}$ denotes the $i, j$ th element of matrix $A$, and $\underline{0}$ denotes the zero vector/matrix.

This paper is concerned with asymptotic mean square stability and reference tracking of nonlinear dynamic systems over AWGN communication channel, as is shown in the block diagram of Figure 1 . The building blocks of the figure are described below.

Dynamic System: The dynamic system is SISO described by the following nonlinear differential equation:

$$
\left\{\begin{array}{l}
f\left(t, x^{(m)}, x^{(m-1)}, \ldots, x, u^{(m)}, u^{(m-1)}, \ldots, u\right)=0 \\
y(t)=x(t)
\end{array}\right.
$$

where $x^{(m)}(t) \in \mathbb{R}$ is the $m$ th derivative of the system output, $u(t) \in \mathbb{R}$ is the control input signal, $u^{(m)}(t) \in \mathbb{R}$ is the $m$ th derivative of the control input signal, and $f\left(t, x^{(m)}, x^{(m-1)}, \ldots, x, u^{(m)}, u^{(m-1)}, \ldots, u\right)=0$ is the arbitrary nonlinear differential equation describing the dynamics of the nonlinear system with the condition that has periodic outputs in response to sinusoidal inputs.

Communication Channel: Communication channel between a system and a controller is an MIMO AWGN channel without interference with $n$ inputs and $n$ outputs. The output of the encoder (which will be described shortly) is transmitted through the MIMO channel and a white Gaussian noise vector is added to it (as is shown in Figure 1), where $N(t)=\left[n_{1}(t) \quad . \quad . \quad . \quad n_{n}(t)\right]^{\prime}$ i.i.d. $\sim N(\underline{0}, \tilde{R})(\tilde{R}=$ $\operatorname{diag}\left\{\tilde{r}_{1}, \ldots, \tilde{r}_{n}\right\} ; \tilde{r}_{i}$ is the variance of the additive noise of the $i$ th input to output path of the channel) is the MIMO channel noise, and $n_{i}(t) \sim N\left(0, \tilde{r}_{i}\right)$ is the additive noise of the $i$ th input to output path of the MIMO AWGN channel. In addition, the MIMO AWGN channel is subject to the channel input power constraint $\left(\mathrm{Po}_{i}, i=1,2, \ldots, n\right)$ as follows:

$$
E\left[f_{i}^{2}(t)\right] \leq P o_{i}, \quad i=1,2, \ldots, n,
$$

where $f_{i}(t)$ is the $i$ th element of the encoder output vector $F(t)=\left[f_{1}(t) \cdot . f_{n}(t)\right]^{\prime}$, which is the input of the channel. Thus, $Y(t)=F(t)+N(t)$, where $Y(t)=\left[y_{1}(t) \quad \cdot \quad \cdot \quad y_{n}(t)\right]^{\prime}$ is the channel output.

Encoder: The encoder is a bandpass filter cascaded with a matrix gain. This bandpass filter saves only the fundamental frequency of system output $y(t)$ and omits the other harmonics which have less information to send. For this purpose, a high-pass filter with a relatively low cut-off frequency (e.g., $0.1 \mathrm{~Hz}$ ) is used to omit the DC part. This filter is cascaded with a low-pass filter and a cut-off frequency of $\omega \gg 2 \pi \times$ $0.1 \mathrm{rad} / \mathrm{s}$. For constructing such a filter, the following transfer functions can be used [33]:

$$
\begin{aligned}
& H_{b p}(s)=H_{h p}(s) H_{l p}(s), \\
& H_{h p}(s)=\frac{s^{2}}{s^{2}+\frac{\omega_{h}}{Q} s+\omega_{h}^{2}}, \quad \omega_{h}=2 \pi \times 0.1 \mathrm{rad} / \mathrm{s}, \\
& Q=\text { damping factor }=0.707 \\
& H_{l p}(s)=\frac{\omega^{2}}{s^{2}+\frac{\omega}{Q} s+\omega^{2}} .
\end{aligned}
$$

It will be shown in the next section that the nonlinear dynamic system (1) together with the bandpass filter has an equivalent linear dynamic system with $n$ states $X(t)=\left[x_{1}(t) \cdot . \quad \cdot x_{n}(t)\right]^{\prime}$, in which these states and matrix gain: 


$$
C(t)=\left[\begin{array}{rrrr}
C_{11}(t) & C_{12}(t) & \ldots & C_{1 n}(t) \\
C_{21}(t) & C_{22}(t) & \ldots & C_{2 n}(t) \\
& \ldots & & \\
C_{n 1}(t) & C_{n 2}(t) & \ldots & C_{n n}(t)
\end{array}\right]
$$

form the encoder output:

$$
F(t)=C(t)(X(t)-\hat{X}(t))
$$

where $\hat{X}(t)=\left[\hat{x}_{1}(t) \cdot . \quad \hat{x}_{n}(t)\right]^{\prime}$ is the estimation of states in the decoder.

Decoder: Decoder is the minimum mean square estimator or the Kalman filter, also known as Linear Quadratic Estimator (LQE) [34]. At each time instant, $t$, the Kalman filter generates an estimation, $\hat{X}(t)$, using channel output $Y(t)$.

Controller: Controller is a certainty equivalent controller [34] of the following form:

$$
u(t)=-L(t) \hat{X}(t)+\nu(t)
$$

The objective of this paper is to find matrix gains $C(t), L(t)$, and $\nu(t)$ to force the states of the linear equivalent system mean square to asymptotically track the reference signal $R(t)=\left[\begin{array}{llll}r_{1}(t) & . & . & r_{n}(t)\end{array}\right]^{\prime}$ where $r_{i}$ is the reference signal of the $i$ th state of the linear equivalent system. Note that one of the states of the equivalent linear system is nonlinear system output $y(t)$; therefore, the corresponding reference signal for this state must be $r(t)$, which is the reference signal of dynamic system output $y(t)$. Moreover, note that stability is a special case of reference tracking with $R(t)=\underline{0}$. Hence, the proposed technique in this paper considers nonlinear system output, $y(t)$, and mean square to facilitate the asymptotic tracking of reference signal, $r(t)$. Now, the exact definition of the reference tracking is given below.

Definition 2.1. Mean square asymptotic tracking of reference signal. Consider the block diagram of Figure 1 described by the nonlinear dynamic system (1) over AWGN channel, as described earlier with the equivalent linear dynamic system with states $X(t)$. It is said that the system uses mean square to asymptotically track reference signal, $R(t)$ (and, consequently, $y(t)$ tracks $r(t)$ ) if there exist an encoder, a decoder, and a controller such that the following property holds for all choices of the initial conditions in spite of the noise and the channel input power constraint.

$$
\begin{aligned}
& \lim _{t \rightarrow \infty} E\left[\left(x_{i}(t)-r_{i}(t)\right)^{2}\right]=0, \\
& \text { subject to } E\left[f_{i}^{2}(t)\right] \leq P o_{i}, \quad \forall i .
\end{aligned}
$$

\section{Implementation of the describing function method}

In this section, the idea of using the describing function to obtain the equivalent linear dynamic system for the nonlinear dynamic system (1) is used. Then, in the next section, an encoder, a decoder, and a controller for mean square asymptotic tracking of the equivalent linear dynamic system, as defined in this section, are proposed.

For all nonlinear dynamic systems that respond periodically to sinusoidal inputs, an equivalent linear dynamic system is found, as defined below [26].

Consider an SISO nonlinear dynamic system with periodic outputs in response to sinusoidal inputs (e.g., system (1)). Suppose that this nonlinear dynamic system is excited by the input: $u(t)=\gamma \cos (\omega t)$, where $\gamma>0$ is large enough to excite all modes of the nonlinear system and $\omega$ is the high cut-off frequency of the filter. Then, since the output is a periodic signal, it has a Fourier series representation [35] that includes all harmonics of the input with a frequency of $\omega$. That is:

$$
\begin{aligned}
& y(t)=y_{d}+\sum_{i=1}^{\infty}\left(a_{i} \sin (i \omega t)+b_{i} \cos (i \omega t)\right), \\
& y_{d}=\frac{\omega}{4 \pi} \int_{-\frac{2 \pi}{\omega}}^{\frac{2 \pi}{\omega}} y(t) d t, \\
& a_{i}=\frac{\omega}{2 \pi} \int_{-\frac{2 \pi}{\omega}}^{\frac{2 \pi}{\omega}}(y(t) \sin (i \omega t)) d t \\
& b_{i}=\frac{\omega}{2 \pi} \int_{-\frac{2 \pi}{\omega}}^{\frac{2 \pi}{\omega}}(y(t) \cos (i \omega t)) d t .
\end{aligned}
$$

Now, if this nonlinear system is cascaded with a bandpass filter and a high cut-off frequency, $\omega$, then we have a periodic output at the end of the filter, which consists of only the first harmonic with frequency $\omega$, and all other harmonics are eliminated. In other words, the output of the bandpass filter is as follows:

$$
y_{f}(t)=a_{1} \sin (\omega t)+b_{1} \cos (\omega t) .
$$

Accordingly, the nonlinear dynamic system that is cascaded with the bandpass filter and the high cutoff frequency of $\omega$ is called a quasi-linear system [26], because we can find a linear dynamic system with input $u(t)=\gamma \cos (\omega t)$ and output $y_{f}(t)$ with the following transfer function:

$$
\begin{aligned}
& H(j \omega)=|H(j \omega)| \angle H(j \omega), \\
& |H(j \omega)|=\frac{\sqrt{a_{1}^{2}+b_{1}^{2}}}{\gamma}, \\
& \angle H(j \omega)=-\arctan \left(\frac{a_{1}}{b_{1}}\right) \mathrm{rad} .
\end{aligned}
$$


This means that the nonlinear dynamic system can be represented by a linear dynamic system with the above transfer function called the describing function of the nonlinear dynamic system. Note that the describing function in $s$ domain can be obtained by assuming the following form of the describing function $H(s)=$ $\frac{1}{s^{2}+\alpha s+\beta}$ (usually, the second-order transfer function is enough to show the behavior of the fundamental frequency of the dynamic system), and, then, determining the real coefficients, $\alpha$ and $\beta$, such that:

$$
|H(j \omega)|=\frac{\sqrt{a_{1}^{2}+b_{1}^{2}}}{\gamma},
$$

and:

$$
\angle H(j \omega)=-\arctan \left(\frac{a_{1}}{b_{1}}\right) \operatorname{rad} .
$$

This describing function represents a linear system with $n=2$ states: $x_{1}(t), x_{2}(t)$, e.g., $x_{1}(t)=y(t)$ and $x_{2}(t)=$ $\dot{y}(t)$ with realization:

$$
\begin{aligned}
& A=\left[\begin{array}{cc}
0 & 1 \\
-\beta & -\alpha
\end{array}\right] \text { and } \\
& B=\left[\begin{array}{l}
0 \\
1
\end{array}\right]
\end{aligned}
$$

one input $u(t)$, and two outputs represented by vector $X(t)=\left[\begin{array}{ll}x_{1}(t) & x_{2}(t)\end{array}\right]^{\prime}$, which is inputted to matrix gain $C(t)$ of the encoder.

For a linear dynamic system with realization ( $A, B)$ where $A$ has only real valued eigenvalues, the mean square stabilization technique over SISO AWGN channel is presented in [10] by finding matrix gains $C(t)$ and $L(t)$. In the next section, first, this result is extended to account for MIMO AWGN channel with a system matrix $A$ with multiple real valued and non-real valued eigenvalues. Then, by applying this extension to the linear equivalent dynamic system, we present the result of the mean square asymptotic tracking of the nonlinear dynamic system (1) with the equivalent linear dynamic system (8) over MIMO AWGN channel.

\section{Linear system with multiple real valued and non-real valued eigenvalues}

Now, the result of [10] is extended to account for the reference tracking and, hence, the stability of linear continuous-time dynamic systems with multiple real valued and non-real valued eigenvalues over the MIMO AWGN channel.

Suppose that the dynamic system in Figure 1 is linear with $n$ states. Hence, the system that is seen by the remote controller is as follows:

$$
\left\{\begin{array}{l}
\dot{X}(t)=A X(t)+B u(t), X(0)=\xi \\
Y(t)=C(t)(X(t)-\hat{X}(t))+N(t)
\end{array}\right.
$$

where $X(0)$ is the initial state, which is unknown for remote controller, $\xi \sim N\left(X_{0}, Q_{0}\right)$ $\left(Q_{0}\right.$ is diagonal) is a random variable, $N(t)=$ $\left[n_{1}(t) \text {. . . } n_{n}(t)\right]^{\prime}$ i.i.d. $\sim N(\underline{0}, \tilde{R}) \quad(\tilde{R}=$ $\left.\operatorname{diag}\left\{\tilde{r}_{1}, \ldots, \tilde{r}_{n}\right\}\right)$ is the additive noise of the MIMO channel, and $\left(n_{i}(t) \sim N\left(0, \tilde{r}_{i}\right)\right.$ is the additive noise of the $i$ th path of the MIMO AWGN channel, which can be treated as the measurement noise provided that the channel input power constraint is met. The first objective in this section is to achieve the mean square asymptotic state tracking, i.e., $E\left[\left(x_{i}(t)-\hat{x}_{i}(t)\right)^{2}\right] \rightarrow 0$, $\forall i$. In this section, we deal with the linear system (11) with $n$ states over MIMO AWGN channel. As discussed in the previous section, this system can represent the state-space realization of the transfer function $H(s)$ (e.g., $A=\left[\begin{array}{cc}0 & 1 \\ -\beta & -\alpha\end{array}\right]$ and $B=\left[\begin{array}{l}0 \\ 1\end{array}\right]$ ), which, in general, includes multiple real valued eigenvalues and non-realvalued eigenvalues.

In this paper, it is assumed that system matrix $A$ has real eigenvalues, real multiple eigenvalues, and distinct complex conjugate eigenvalues, since system matrix $A$ that corresponds to transfer function $H(s)$ includes these types of eigenvalues. Without loss of generality, we can always assume that system matrix $A$ is in the real Jordan form [8]. This form is obtained by implementing a proper similarity transformation [36]; as a result of that, system (11) is decomposed into several decoupled sub-systems, in which, an encoder and a decoder can be designed separately for the state tracking for each sub-system. Note that the Jordan block associated with a real eigenvalue $\lambda_{i}(A)$ with multiplicity $d_{i}$ is the following matrix:

$$
\left[\begin{array}{cccccccc}
\lambda_{i}(A) & a & 0 & . & . & . & 0 & 0 \\
0 & \lambda_{i}(A) & b & . & . & . & 0 & 0 \\
\cdot & & & & & & & \\
\cdot & & & & & & & \\
\cdot & & & & & & & \\
0 & 0 & 0 & . & . & . & \lambda_{i}(A) & c \\
0 & 0 & 0 & . & . & . & 0 & \lambda_{i}(A)
\end{array}\right]
$$

where $a, b, c \in\{0,1\}$ depends on the rank of matrix $\left(\lambda_{i}(A) I_{n}-A\right)$. In addition, note that the Jordan block associated with the complex conjugate pair of the eigenvalues $\lambda_{i}(A)=\sigma \pm \sqrt{-1} \omega(\omega \neq 0)$ is $\left[\begin{array}{cc}\sigma & \omega \\ -\omega & \sigma\end{array}\right]$.

\subsection{Mean square asymptotic state tracking}

In this section, it is assumed that each of the Jordan block is at most a 2-by-2 matrix (the general case can be treated similarly). Then, for all three following possible cases:
(a) $A=\left[\begin{array}{cc}a_{1} & 0 \\ 0 & a_{2}\end{array}\right], a_{1}, a_{2} \in \mathbb{R}, a_{1} \neq a_{2}$,
(b) $A=\left[\begin{array}{cc}a & 1 \\ 0 & a\end{array}\right], \quad a \in \mathbb{R}$, 
(c) $A=\left[\begin{array}{cc}\sigma & \omega \\ -\omega & \sigma\end{array}\right], \quad \sigma, \omega \in \mathbb{R}, \quad \omega \neq 0$,

we find matrix gain $C(t)$ for mean square asymptotic tracking of system states at the decoder.

4.1.1. Sub-system with real distinct eigenvalues

Suppose that system matrix $A$ in the linear system of (11) has the following form:

$$
A=\left[\begin{array}{lr}
a_{1} & 0 \\
0 & a_{2}
\end{array}\right], \quad a_{1}, a_{2} \in \mathbb{R}, \quad a_{1} \neq a_{2} .
$$

Then, we have the following proposition for the mean square asymptotic tracking of system states at the decoder.

Proposition 4.1. Consider the block diagram of Figure 1 described by the linear system (11) with the system matrix $A=\left[\begin{array}{cc}a_{1} & 0 \\ 0 & a_{2}\end{array}\right], a_{1}, a_{2} \in \mathbb{R}, a_{1} \neq a_{2}$. Suppose that the pair $(A, B)$ is controllable and $P_{o_{1}}>$ $2 a_{1}$ and $\mathrm{Po}_{2}>2 a_{2}\left(\mathrm{Po}_{1}\right.$ and $\mathrm{Po}_{2}$ are the channel input power constraints). Then, by choosing the following encoder matrix gain:

$$
C(t)=\left[\begin{array}{lr}
\sqrt{\frac{P o_{1} \tilde{r}_{1}}{P_{11}(t)}} & 0 \\
0 & \sqrt{\frac{P o_{2} \tilde{r}_{2}}{P_{22}(t)}}
\end{array}\right],
$$

and a decoder with the following description (which is the Kalman filter [34])

$$
\begin{aligned}
& \dot{\hat{X}}(t)=A \hat{X}(t)+B u(t)+K(t) Y(t), \quad \hat{X}(0)=X_{0}, \\
& K(t)=P(t) C^{\prime}(t) \tilde{R}^{-1}, \tilde{R}=\operatorname{diag}\left\{\tilde{r}_{1}, \tilde{r}_{2}\right\}, \\
& \dot{P}(t)=A^{\prime} P(t)+P(t) A-P(t) C^{\prime}(t) \tilde{R}^{-1} C(t) P(t), \\
& P(0)=Q_{0}, \quad P(t)=P^{\prime}(t)=\left[\begin{array}{ll}
P_{11}(t) & P_{12}(t) \\
P_{12}(t) & P_{22}(t)
\end{array}\right] \geq 0,
\end{aligned}
$$

we have the mean square asymptotic tracking of system states at the decoder.

Proof: The encoder matrix gain has the following general form:

$$
C(t)=\left[\begin{array}{ll}
C_{11}(t) & C_{12}(t) \\
C_{21}(t) & C_{22}(t)
\end{array}\right]
$$

Subsequently, the decoder can be extracted as follows (for the simplicity of presentation, the dependency on time index $t$ is dropped):

$$
\left\{\begin{aligned}
\dot{P}_{11} & =2 a_{1} P_{11}-\left(2 P_{11} P_{12} C_{11} C_{12} \tilde{r}_{1}^{-1}\right. \\
& +2 P_{11} P_{12} C_{21} C_{22} \tilde{r}_{2}^{-1}+P_{11}^{2} C_{11}^{2} \tilde{r}_{1}^{-1} \\
& \left.+P_{12}^{2} C_{12}^{2} \tilde{r}_{1}^{-1}+P_{11}^{2} C_{21}^{2} \tilde{r}_{2}^{-1}+P_{12}^{2} C_{22}^{2} \tilde{r}_{2}^{-1}\right) \\
\dot{P_{22}} & =2 a_{2} P_{22}-\left(2 P_{22} P_{12} C_{22} C_{21} \tilde{r}_{2}^{-1}\right. \\
& +2 P_{22} P_{12} C_{21} C_{11} \tilde{r}_{1}^{-1}+P_{22}^{2} C_{22}^{2} \tilde{r}_{2}^{-1} \\
& \left.+P_{12}^{2} C_{21}^{2} \tilde{r}_{2}^{-1}+P_{22}^{2} C_{12}^{2} \tilde{r}_{1}^{-1}+P_{12}^{2} C_{11}^{2} \tilde{r}_{1}^{-1}\right) \\
& \dot{P} \\
P_{12} & =a_{1} P_{12}+a_{2} P_{12}-\tilde{r}_{1}^{-1}\left(P_{11} P_{12} C_{11}^{2}\right. \\
& \left.+P_{12}^{2} C_{11} C_{12}+P_{11} P_{22} C_{11} C_{12}+P_{12} P_{22} C_{12}^{2}\right) \\
& -\tilde{r}_{2}^{-1}\left(P_{11} P_{12} C_{21}^{2}+P_{12}^{2} C_{22} C_{21}+P_{11} P_{22} C_{22} C_{21}\right. \\
& \left.+P_{12} P_{22} C_{22}^{2}\right)
\end{aligned}\right.
$$

Now, by substituting $C_{11}(t)=\sqrt{\frac{P_{0} \tilde{r}_{1}}{P_{11}(t)}}, C_{22}(t)=$ $\sqrt{\frac{P o_{2} \tilde{r}_{2}}{P_{22}(t)}}$ and $C_{12}(t)=C_{21}(t)=0$ in the above expressions, we have $\dot{P}_{12}(t)=0$ and $P_{12}(t)=P_{12}(0)=$ $\left[Q_{0}\right]_{12}=0$. Thus, we have:

$$
\begin{aligned}
\dot{P}_{11}(t) & =\left(2 a_{1}-P o_{1}\right) P_{11}(t) \Rightarrow P_{11}(t) \\
& =e^{-\left(P o_{1}-2 a_{1}\right) t} P_{11}(0), \\
\dot{P}_{22}(t) & =\left(2 a_{2}-P o_{2}\right) P_{22}(t) \Rightarrow P_{22}(t) \\
& =e^{-\left(P o_{2}-2 a_{2}\right) t} P_{22}(0) .
\end{aligned}
$$

In summary, through the above selection, we have:

$$
P(t)=\left[\begin{array}{lr}
e^{-\left(P o_{1}-2 a_{1}\right) t} P_{11}(0) & 0 \\
0 & e^{-\left(P o_{2}-2 a_{2}\right) t} P_{22}(0)
\end{array}\right],
$$

and the following description for the decoder:

$$
\begin{aligned}
& \dot{\hat{X}}(t)=A \hat{X}(t)+B u(t)+K(t) Y(t), \\
& K(t)=P(t) C^{\prime}(t) \tilde{R}^{-1}, \quad \hat{X}(0)=X_{0} .
\end{aligned}
$$

Now, it is assumed that $\mathrm{Po}_{1}>2 a_{1}$ and $\mathrm{Po}_{2}>2 a_{2}$; accordingly, we have:

$$
\begin{gathered}
\lim _{t \rightarrow \infty} E\left[\left(x_{1}(t)-\hat{x}_{1}(t)\right)^{2}\right]=\lim _{t \rightarrow \infty} P_{11}(t) \\
=\lim _{t \rightarrow \infty} e^{-\left(P o_{1}-2 a_{1}\right) t} P_{11}(0)=0 .
\end{gathered}
$$

Similarly, we have:

$$
\lim _{t \rightarrow \infty} E\left[\left(x_{2}(t)-\hat{x}_{2}(t)\right)^{2}\right]=\lim _{t \rightarrow \infty} P_{22}(t)=0 .
$$

Hence:

$$
P(t) \rightarrow \bar{P}=\left[\begin{array}{ll}
0 & 0 \\
0 & 0
\end{array}\right] .
$$

This completes the proof. 
4.1.2. Sub-system with real multiple eigenvalues

Suppose that system matrix $A$ in the linear system of (11) has the following form:

$$
A=\left[\begin{array}{ll}
a & 1 \\
0 & a
\end{array}\right], \quad a \in \mathbb{R}
$$

Then, we have the following proposition for mean square asymptotic tracking of system states at the decoder.

Proposition 4.2. Consider the block diagram of Figure 1 described by the linear system (11) with system matrix:

$$
A=\left[\begin{array}{cc}
a & 1 \\
0 & a
\end{array}\right], \quad a \in \mathbb{R} .
$$

Suppose that the pair $(A, B)$ is controllable, $P_{o_{1}}>2 a$ $\mathrm{Po}_{2}>2 a,\left[Q_{0}\right]_{12}=0,\left[Q_{0}\right]_{11}=\left[Q_{0}\right]_{22}$, and $\tilde{r}_{1}=\tilde{r}_{2}$. Then, by choosing the encoder matrix gain as:

$$
C(t)=\left(\begin{array}{ll}
\sqrt{\frac{\tilde{r}_{1}}{2 \delta_{P 11}(t)}} & \sqrt{\frac{\delta \tilde{r}_{1}}{22_{P 22}(t)}} \\
\sqrt{\frac{\delta \tilde{r}_{2}}{2 P_{P 11}(t)}} & \sqrt{\frac{\tilde{r}_{2}}{2 \delta_{P 22}(t)}}
\end{array}\right)
$$

where $\delta=\gamma_{1}-\sqrt{\gamma_{1}^{2}-1}$ and $\gamma_{1}=\min \left(P_{o_{1}}, P_{O_{2}}\right)$, and the decoder (which is the Kalman filter [34]) as:

$$
\begin{aligned}
& \dot{\hat{X}}(t)=A \hat{X}(t)+B u(t)+K(t) Y(t), \quad \hat{X}(0)=X_{0}, \\
& K(t)=P(t) C^{\prime}(t) \tilde{R}^{-1}, \tilde{R}=\operatorname{diag}\left\{\tilde{r}_{1}, \tilde{r}_{2}\right\} \\
& \dot{P}(t)=A^{\prime} P(t)+P(t) A-P(t) C^{\prime}(t) \tilde{R}^{-1} C(t) P(t), \\
& P(0)=Q_{0}, P(t)=P^{\prime}(t)=\left[\begin{array}{ll}
P_{11}(t) & P_{12}(t) \\
P_{12}(t) & P_{22}(t)
\end{array}\right] \geq 0,
\end{aligned}
$$

we have the mean square asymptotic tracking of system states at the decoder.

Proof: It is similar to the proof of Proposition 4.1.

4.1.3. Sub-system with complex conjugate eigenvalues Suppose that the system matrix $A$ in the linear system of (11) has the following form:

$$
A=\left[\begin{array}{cc}
\sigma & \omega \\
-\omega & \sigma
\end{array}\right], \quad \sigma, \omega \in \mathbb{R}, \quad \omega \neq 0
$$

Then, we have the following proposition for mean square asymptotic tracking of system states at the decoder.
Proposition 4.3. Consider the block diagram of Figure 1 described by the linear system (11) with the system matrix:

$$
A=\left[\begin{array}{cc}
\sigma & \omega \\
-\omega & \sigma
\end{array}\right], \quad \sigma, \omega \in \mathbb{R}, \quad \omega \neq 0 .
$$

Suppose that the pair $(A, B)$ is controllable, $P_{o_{1}}>2 \sigma$ and $P_{o_{2}}>2 \sigma,\left[Q_{0}\right]_{12}=0,\left[Q_{0}\right]_{11}=\left[Q_{0}\right]_{22}$, and $\tilde{r}_{1}=$ $\tilde{r}_{2}$. Then, by choosing the encoder matrix gain as:

$$
C(t)=\left(\begin{array}{rr}
\sqrt{\frac{\gamma_{1} \tilde{r}_{1}}{p_{11}(t)}} & 0 \\
0 & \sqrt{\frac{\gamma_{1} \tilde{r}_{2}}{p_{22}(t)}}
\end{array}\right),
$$

and the decoder (which is the Kalman filter [34]) as:

$$
\begin{aligned}
& \dot{\hat{X}}(t)=A \hat{X}(t)+B u(t)+K(t) Y(t), \quad \hat{X}(0)=X_{0}, \\
& K(t)=P(t) C^{\prime}(t) \tilde{R}^{-1}, \quad \tilde{R}=\operatorname{diag}\left\{\tilde{r}_{1}, \tilde{r}_{2}\right\}, \\
& \dot{P}(t)=A^{\prime} P(t)+P(t) A-P(t) C^{\prime}(t) \tilde{R}^{-1} C(t) P(t), \\
& P(0)=Q_{0}, \quad P(t)=P^{\prime}(t)=\left[\begin{array}{ll}
P_{11}(t) & P_{12}(t) \\
P_{12}(t) & P_{22}(t)
\end{array}\right] \geq 0,
\end{aligned}
$$

we have the mean square asymptotic tracking of system states at the decoder.

Proof: It is similar to the proof of Proposition 4.1.

\subsection{Asymptotic reference tracking}

Now, to obtain the control signal for tracking reference signal $R(t)$, consider the following cost function:

$$
\begin{aligned}
J= & \lim _{t_{1} \rightarrow \infty} \frac{1}{t_{1}} \int_{0}^{t_{1}} E\left[[X(t)-R(t)]^{\prime} Q[X(t)-R(t)]\right. \\
& \left.+\rho u^{2}(t)\right] d t, \quad Q=Q^{\prime} \geq 0, \quad \rho>0 .
\end{aligned}
$$

Control signal $u(t)$ is obtained by minimizing the above cost function which is subject to the dynamic system (11). Based on [34], $u(t)=-L(t) \hat{X}(t)+\nu(t)$, where:

$$
\begin{aligned}
& L(t)=\rho^{-1} B^{\prime} \tilde{P}(t), \\
& \nu(t)=-\rho^{-1} B^{\prime} S(t),
\end{aligned}
$$

and $\tilde{P}(t)$ and $S(t)$ are the results of the following equations ( $Q$ and $\rho$ are arbitrary):

$$
\begin{aligned}
& \dot{\tilde{P}}(t)=-\tilde{P}(t) A-A^{\prime} \tilde{P}(t)-Q+\tilde{P}(t) B \rho^{-1} B^{\prime} \tilde{P}(t), \\
& \dot{S}(t)=-\left[A^{\prime}-\tilde{P}(t) B \rho^{-1} B^{\prime}\right] S(t)+Q R(t) .
\end{aligned}
$$

Under the assumption that the pair $(A, B)$ is controllable, we have $\tilde{P}(t) \rightarrow \overline{\tilde{P}}[34]$; hence, $J \rightarrow \bar{J}=0$. This indicates that:

$$
E[X(t)-R(t)]^{\prime} Q[X(t)-R(t)] \rightarrow 0 .
$$

Hence, $E\left[\left(x_{i}(t)-r_{i}(t)\right)^{2}\right] \rightarrow 0, \forall i$ (if matrix $Q$ is diagonal). 


\section{Reference tracking of the nonlinear dynamic system}

In this section, we have the following proposition for mean square asymptotic reference tracking of the nonlinear dynamic system (1) (with the equivalent linear dynamic system (8)) in the block diagram of Figure 1.

Proposition 5.1. Consider the control system of Figure 1 described by the nonlinear dynamic system (1) over MIMO AWGN channel subject to the power constraint $E\left[f_{i}^{2}(t)\right] \leq P o_{i}$, as described earlier. Suppose that the state-space realization $(A(\omega), B(\omega))$ for transfer function $H(s)$ is controllable. Then, there exist an encoder, a decoder, and a controller that force the state mean square to track asymptotically reference signal $R(t)$; hence, nonlinear dynamic system output $y(t)$ of mean square asymptotically tracks reference signal $r(t)$.

Proof: In Section 3, $H(s)=\frac{1}{s^{2}+\alpha s+\beta}$; hence, the state-space realization of $H(s)$ is $(A(\omega), B(\omega))$ with $n=2$. Subsequently, encoder matrix gain $C(t)$ and the decoder are determined through Propositions 4.14.3 ; hence, according to the results of Section 4.2 , the controller of the form of $u(t)=-L(t) \hat{X}(t)+\nu(t)$ is represented, where $L(t)$ and $\nu(t)$, obtained respectively through Eq. (20) and (21), result in the reference tracking. In other words, by using this control policy, the output of the bandpass filter minus the reference signal, i.e., $X(t)-R(t)$, converges to zero in the mean square sense (see Figure 2). Note that one of the elements of vector $X(t)$ (i.e., the state vector of the realization of the describing function) is the system output with the DC part excluded (due to the bandpass filter). Hence, although it is expected that $y(t)-r(t) \rightarrow$ 0 (as $X(t)-R(t) \rightarrow 0$ ), the designed control signal may make $y(t)-r(t)$ converge to a non-zero value. Therefore, to make $y(t)-r(t)$ also converge to zero, the inner control loop with gain $\frac{k}{s}$ (where $k$ is large enough, e.g., 25) is used to attenuate the effect of the DC bias in signal $y(t)-r(t)$ as much as possible so that $y(t)$ also tracks $r(t)$. This completes the proof.

\section{Simulation results}

In this section, for the purpose of illustration, we apply the proposed an encoder, a decoder, and a controller to a DC motor with a saturation element, used in our laboratory, to protect the DC motor from high input voltage, as is shown in Figure 3. Systems with an actuator saturation and hard constraints represent an important class of dynamic systems, which have been considered in the literature (e.g., [37-39]). The DC motor considered in this paper has the following description:

$$
\left\{\begin{array}{l}
\dot{x}(t)=-0.96 x(t)+178.85 \tilde{u}(t) \\
y(t)=x(t)
\end{array}\right.
$$

where $\tilde{u}(t)$ is the output of the saturation element. The saturation element saturates control signal $u(t)$ between -10 and +10 volts. That is:

$$
\tilde{u}(t)=\left\{\begin{array}{l}
10, u(t) \geq 10 \\
u(t),|u(t)|<10 \\
-10, u(t) \leq-10
\end{array}\right.
$$

For the remote controller, the initial condition is unknown and has the description of $x(0) \sim N(0,1)$. The system must track reference signal $r(t)$. The MIMO AWGN channel has the specification of $N(t)$ i.i.d. $N(\underline{0}, \operatorname{diag}\{1,1\})$, with the power constraints of $P_{o_{1}}=2$ and $\mathrm{PO}_{2}=2$ to satisfy the requirements of Propositions 4.1-4.3.

The linear dynamic system (24) can be treated as a low-pass filter, which is cascaded with the nonlinear saturation block (see Figure 3). Hence, this block together with the linear dynamic system (24) has a

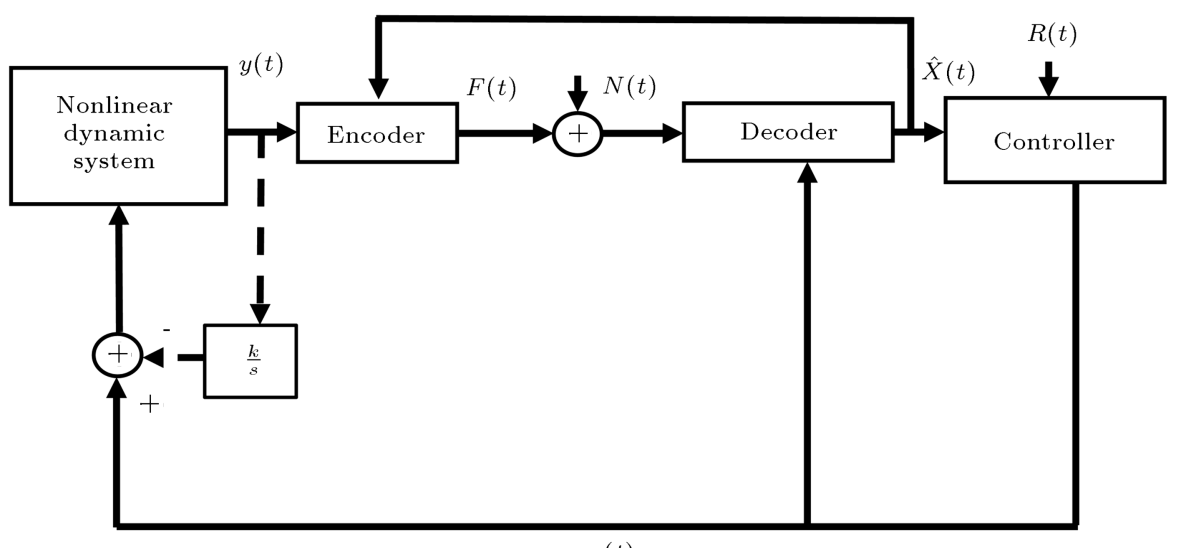

$u(t)$

Figure 2. Nonlinear dynamic system (1) controlled over AWGN channel. 


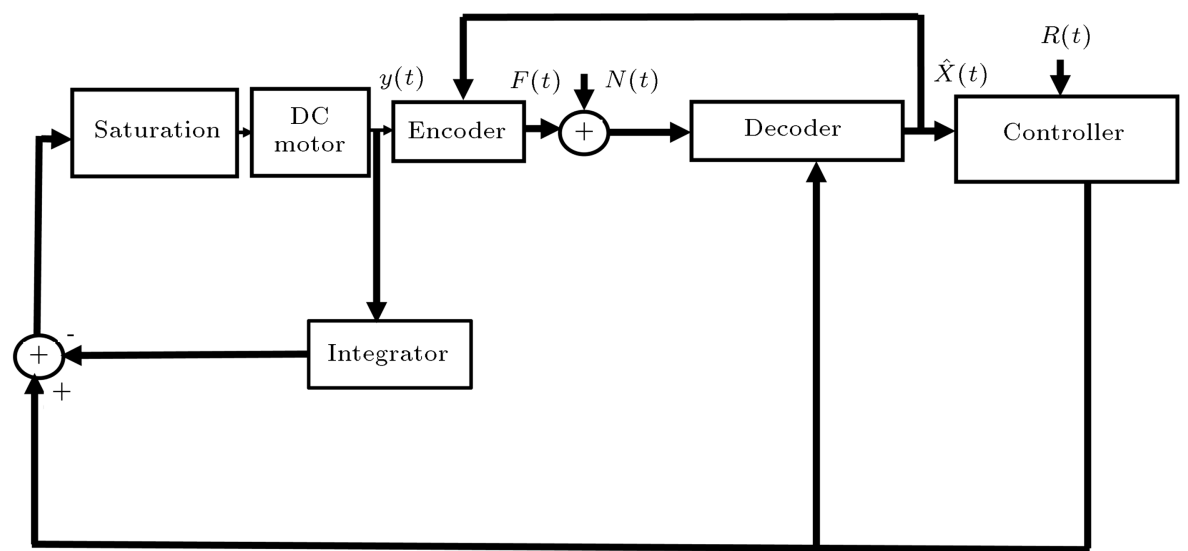

$u(t)$

Figure 3. A nonlinear dynamic system controlled over AWGN channel.

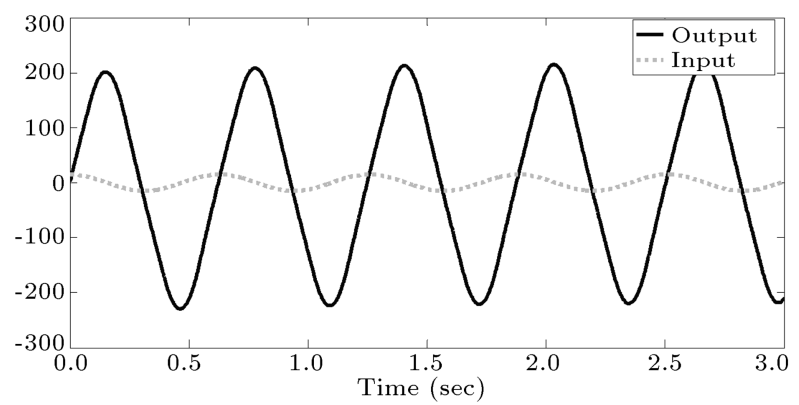

Figure 4. The output of the nonlinear system for sinusoidal input $u(t)=15 \cos (10 t)$ and $x(0)=1$.

quasi-linear representation described by the describing function. To obtain this describing function for a given $\omega$, e.g., $\omega=10$, the following sinusoidal input is applied to the system:

$$
u(t)=15 \cos (10 t) .
$$

The steady-state output of this excitation signal is:

$$
y(t)=x(t)=208.5 \cos (10 t+1.67) \mathrm{rad} / \mathrm{s},
$$

which is shown in Figure 4. Based on the input and output, the describing function is:

$$
H(s)=\frac{1}{s^{2}+0.00716 s+99.99},
$$

therefore, the equivalent linear dynamic system that is seen by the remote controller in the Jordan form is presented in the following:

$$
\left\{\begin{array}{l}
\dot{X}(t)=\left[\begin{array}{cc}
-0.0036 & -9.9996 \\
9.9996 & -0.0036
\end{array}\right] X(t)+\left[\begin{array}{c}
0 \\
-0.1
\end{array}\right] u(t) \\
y(t)=\left[\begin{array}{ll}
1 & 0
\end{array}\right] X(t) \\
Y(t)=C(t)(X(t)-\hat{X}(t))+N(t)
\end{array}\right.
$$

Note that $X_{1}(t)=y(t)$; therefore, $r_{1}(t)=r(t)$. In addition, the encoder is described by matrix gain
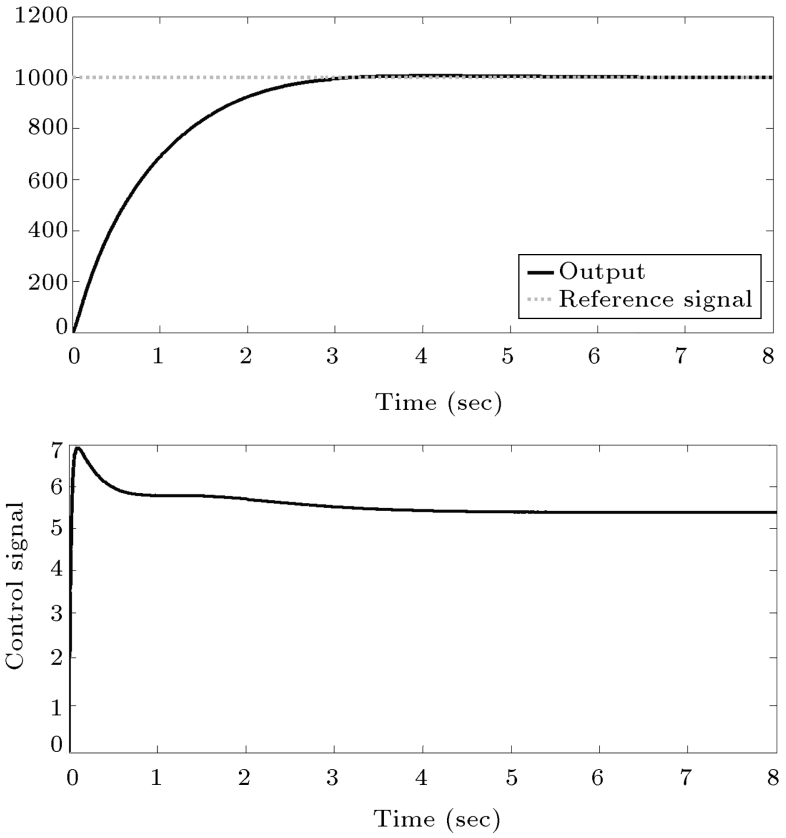

Figure 5. The response of the system and control signal for $r(t)=1000 \mathrm{rad} / \mathrm{s}$.

$C(t)$. Now, by applying the previous theoretical developments to this system, we have the mean square asymptotic tracking, as follows.

Figure 5 illustrates system output $y(t)$ and control signal $u(t)$ for $r(t)=1000 \mathrm{rad} / \mathrm{s}$. Figures 6 and 7 illustrate system output $y(t)$ and control signal $u(t)$ for $r(t)=1000 \cos (0.2 t) \mathrm{rad} / \mathrm{s}$ and $r(t)=$ $2000 \cos (0.2 t) \mathrm{rad} / \mathrm{s}$, respectively. As is clear from these figures, the proposed reference tracking technique is able to track the reference signal with a suitable transient response.

To magnify the performance of the proposed technique, we also applied the method of [10], which is for a linear system, to the system (24) without considering the saturation in the design of control signal 

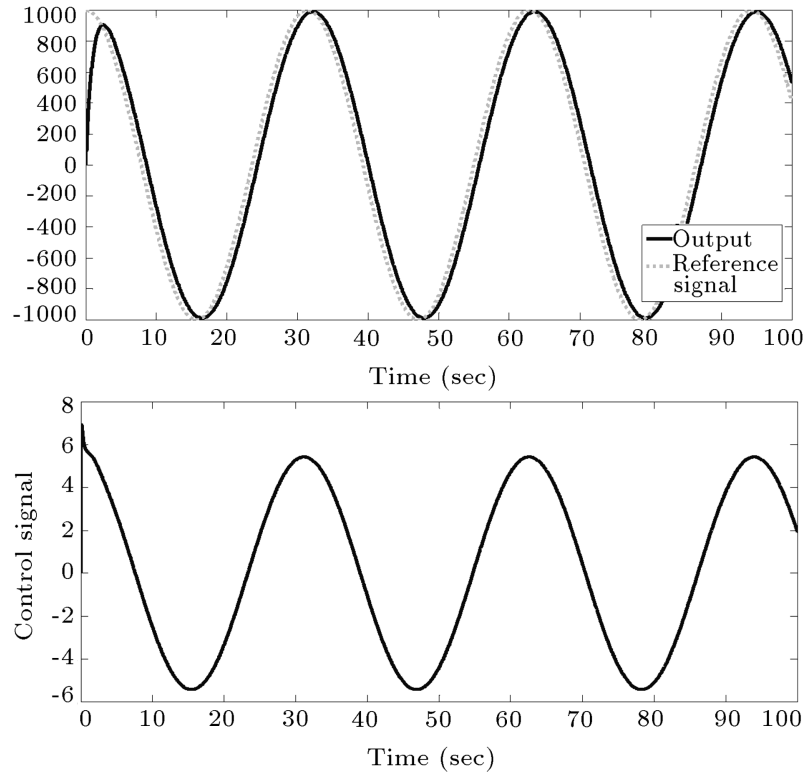

Figure 6. The response of the system and control signal for $r(t)=1000 \cos (0.2 t) \mathrm{rad} / \mathrm{s}$.
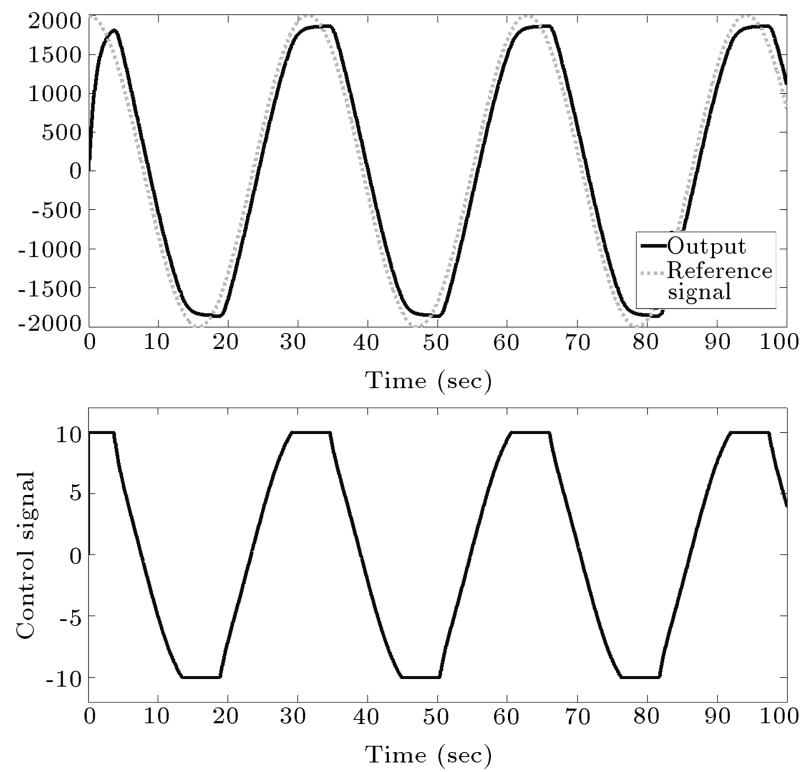

Figure 7. The response of the system and control signal for $r(t)=2000 \cos (0.2 t) \mathrm{rad} / \mathrm{s}$.

(as the method proposed in [10] is only considered for linear systems). Figure 8 illustrates the simulation results of this case when the reference signal is $r(t)=$ $2000 \cos (0.2 t) \mathrm{rad} / \mathrm{s}$. A comparison of the results of Figures 7 and 8 illustrates that our proposed technique has a better performance.

\section{Conclusion and direction for future research}

In this paper, a new technique for mean square asymptotic reference tracking of nonlinear dynamic systems over AWGN channel was presented. To achieve
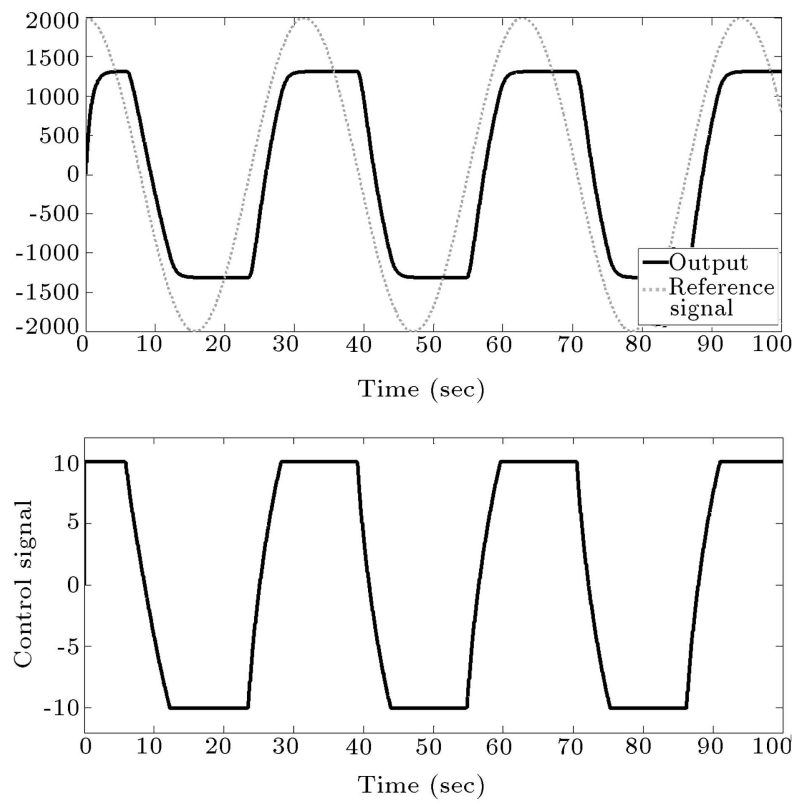

Figure 8. The response of the system and control signal of the linear method of [10] for $r(t)=2000 \cos (0.2 t) \mathrm{rad} / \mathrm{s}$.

this goal, by using the describing function method, an equivalent linear dynamic system was extracted for a nonlinear dynamic system that has periodic outputs and sinusoidal inputs and was cascaded with a bandpass filter acting as an encoder. Then, the results of [10] were extended to account for the reference tracking of continuous-time systems with multiple real valued and non-real valued eigenvalues over MIMO AWGN channel. Then, by applying these extended results to the equivalent linear dynamic system, a new technique for mean square asymptotic reference tracking of nonlinear dynamic systems over AWGN channel was presented. The proposed technique for mean square asymptotic reference tracking includes an encoder consisting of a bandpass filter cascaded with a matrix gain, the Kalman filter [34], and a certainty equivalent controller [34]. The satisfactory performance of the proposed technique was illustrated by computer simulations.

For the future, it is interesting to find techniques for reference tracking of the other class of nonlinear dynamic systems over the AWGN channel. The application of the proposed technique to the nonlinear unicycle model [1], which represents the dynamic of the autonomous vehicles, is currently under investigations by the authors.

\section{References}

1. Farhadi, A., Domun, J., and Canudas de Wit, C. "A supervisory control policy over an acoustic communication network", International Journal of Control, 88(5), pp. 946-958 (2015). 
2. Memarzadeh, M., Optimal Borehole Communication Using Multicarrier Modulation, Diss. Rice University (2007).

3. Elia, N. "When Bode meets Shannon: control-oriented feedback communication schemes", IEEE Trans. Automat. Contr., 49(9), pp. 1477-1488 (2004).

4. Martins, N.C., Dahleh, A., and Elia N. "Feedback stabilization of uncertain systems in the presence of a direct link", IEEE Trans. Automat. Contr., 51(3), pp. $438-447$ (2006).

5. Minero, P., Coviello, L., and Franceschetti, M. "Stabilization over Markov feedback channels: the general case", IEEE Trans. Automat. Contr., 58(2), pp. 349362 (2013).

6. Nair, G.N., Evans, R.J., Mareels, I.M.Y., and Moran, W. "Topological feedback entropy and nonlinear stabilization"', IEEE Trans. Automat. Contr., 49(9), pp. 1585-1597 (2004).

7. Nair, G.N. and Evans, R.J. "Stabilizability of stochastic linear systems with finite feedback data rates", SIAM J. Control Optimization, 43(3), pp. 413-436 (2004).

8. Tatikonda, S. and Mitter, S. "Control under communication constraints", IEEE Transactions on Automatic Control, 49(7), pp. 1056-1068 (2004).

9. Charalambous, C.D. and Farhadi, A. "LQG optimality and separation principle for general discrete time partially observed stochastic systems over finite capacity communication channels", Automatica, 44(12), pp. 3181-3188 (2008).

10. Charalambous, C.D., Farhadi, A., and Denic, S.Z. "Control of continuous-time linear Gaussian systems over additive Gaussian wireless fading channels: a separation principle", IEEE Trans. Automat. Contr., 53(4), pp. 1013-1019 (2008).

11. Farhadi, A. "Stability of linear dynamic systems over the packet erasure channel: a co-design approach", International Journal of Control, 88(12), pp. 24882498 (2015).

12. Farhadi, A. "Feedback channel in linear noiseless dynamic systems controlled over the packet erasure network", International Journal of Control, 88(8), pp. 1490-1503 (2015).

13. Minero, P., Franceschetti, M., Dey, S., and Nair, G.N. "Data rate theorem for stabilization over time-varying feedback channels", IEEE Trans. Automat. Contr., 54(2), pp. 243-255 (2009).

14. Elia, N. and Eisenbeis, J.N. "Limitations of linear control over packet drop networks", IEEE Trans. Automat. Contr., 56(4), pp. 826-841 (2011).

15. Canudas de Wit, C., Gomez-Estern, F., and Rodrigues Rubio, F. "Delta-modulation coding redesign for feedback-controlled systems", IEEE Transactions on Industrial Electronics, 56(7), pp. 2684-2696 (2009).

16. Braslavsky, J.H., Middleton, R.H., and Freudenberg, J.S. "Feedback stabilisation over signal-to-noise ratio constrained channels", IEEE Transactions on Automatic Control, 52(8), pp. 1391-1403 (2007).

17. Farhadi, A. and Ahmed, N.U. "Suboptimal decentralized control over noisy communication channels", Systems and Control Letters, 60, pp. 282-293 (2011).

18. Farhadi, A. and Charalambous, C.D. "Stability and reliable data reconstruction of uncertain dynamic systems over finite capacity channels", Automatica, 46(5), pp. 889-896 (2010).

19. Najafi, S. M. and Farhadi, A. "Stability and tracking of linear Gaussian systems over AWGN channel with intermittent deterministic feedback channel", in The Proceedings of the 23rd Iranian Conference on Electrical Engineering, Tehran, Iran (2015).

20. Li, Y., Chen, J., Tuncel, E., and Su, W. "MIMO control over additive white noise channels: stabilization and tracking by LTI controllers", IEEE Transactions on Automatic Control, 61(5), pp. 1281-1296 (2016).

21. Wu, J. and Chen, T. "Design of networked control systems with packet dropouts", IEEE Transactions on Automatic Control, 52(7), pp. 1314-1319 (2007).

22. Cover, T.A. and Thomas, J.A., Elements of Information Theory, John Wiley and Sons (1991).

23. Judd, K.L., Numerical Methods in Economics, Cambridge, MA: MIT Press (1998).

24. Mees, A.I., Dynamics of Feedback Systems, New York: Wiley (1981).

25. Gilmore, R.J. and Steer, M.B. "Nonlinear circuit analysis using the method of harmonic balance A review of the art. Part I. Introductory concepts", Int. J. Microw. Mill.-Wave Comput.-Aided Eng., 1, pp. 2227 (1991).

26. Slotine, J.J. and Li, W., Applied Nonlinear Control, Prentice-Hall (1991).

27. Jing, Xi. and Lang, Zi., Frequency Domain Analysis and Design of Nonlinear Systems Based on Volterra Series Expansion - A Parametric Characteristic Approach, Springer International Publishing Switzerland (2015).

28. Rugh, W.J., Nonlinear System Theory: the Volterra/Wiener Approach, Baltimore, MD: The Johns Hopkins Univ. Press (1981).

29. Worden, K. and Tomlinson, G.R., Nonlinearity in Structural Dynamics: Detection, Identification and Modeling, Bristol, U.K.: Institute of Physics (2001). 
30. George, D.A., Continuous Nonlinear Systems, MIT Research Lab. Electronics, Cambridge, MA, Tech. Rep. 355 (1959).

31. Xiao, Z. and Jing, X. "Frequency-domain analysis and design of linear feedback of nonlinear systems and applications in vehicle suspensions", IEEE/ASME Transactions on Mechatronics, 21(1), pp. 506-517 (2016).

32. Jing, X. "Nonlinear characteristic output spectrum for nonlinear analysis and design", IEEE/ASME Transactions on Mechatronics, 19(1), pp. 171-183 (2014).

33. Shenoi, B.A., Introduction to Digital Signal Processing and Filter Design, John Wiley and Sons (2006).

34. Kwakernaak, H. and Sivan, P., Linear Optimal Control Systems, Wiley-Interscience (1972).

35. Rudin, W., Principle of Mathematical Analysis, New York: McGraw - Hill, Inc. (1976).

36. Strang, G., Introduction to Linear Algebra, Wellesley Cambridge Press (2016).

37. Pan, H., Sun, W., Gao, H., and Jing, X. "Disturbance observer-based adaptive tracking control with actuator saturation and its application", IEEE Transactions on Automation Science and Engineering, 13(2), pp. 868875 (2016).

38. Pan, H., Sun, W., Gao, H., and Yu, J. "Finite-time stabilization for vehicle active suspension systems with hard constraints", IEEE Transactions on Intelligent Transportation Systems 16(5), pp. 2663-2672 (2015).

39. Sun, W., Pan, H., and Gao, H. "Filter-based adaptive vibration control for active vehicle suspensions with electrohydraulic actuators", IEEE Transactions on Vehicular Technology, 65(6), pp. 4619-4626 (2016).

\section{Biographies}

Ali Parsa is currently a PhD student at the Department of Electrical Engineering at Sharif University of Technology. His research is concerned with control over communication. He received his BSc and MSc degrees in Electrical Engineering from the University of Tehran, Iran.

Alireza Farhadi received $\mathrm{PhD}$ degree in Electrical Engineering from the University of Ottawa, Ontario, Canada in 2007. After receiving PhD degree, Dr. Farhadi worked as a Postdoctoral Fellow at the Department of Electrical Engineering of the University of Ottawa (2008-2009) and the French National Institute for Research in Computer Science and Control (INRIA), Grenoble, France (2010-2011). He then worked as a Research Fellow (academic level B) at the Department of Electrical Engineering of the University of Melbourne, Australia (2011-2013). In September 2013, he joined the Department of Electrical Engineering of the Sharif University of Technology as an Assistant Professor. Dr. Farhadi's areas of expertise include networked control systems, distributed optimal control, stochastic and nonlinear controls, automated irrigation networks, and IoT. 